\title{
Valoración de una dieta hipercalórica en el desarrollo de osteoporosis en ratas Sprague-Dawley gonadectomizadas
}

\author{
Mónica A. Gutiérrez¹, Giovanna Rincón' ${ }^{1}$ Antonio J. Bermúdez²
}

\section{Resumen}

El trabajo se inició con 34 ratas Sprague-Dawley de siete semanas de edad, de las cuales 26 se sometieron a gonadectomía bilateral para inducirles la menopausia. EI grupo control quedó constituido por las restantes 8 ratas no gonadectomizadas. Para evaluar la influencia de la dieta hipercalórica en el desarrollo de osteoporosis, a 13 ratas gonadectomizadas se les suministró una dieta rica en grasa (grupo A), mientras que las otras 13 ratas (grupo B) y el grupo control recibieron una dieta regular.

Cinco meses después de la ovariectomía, la dieta hipercalórica causó la disminución del contenido de calcio en vértebras lumbares ( $L 3, L 4$ y L5), mostrándose el efecto de la dieta en cuanto a que, bajo la ingesta de los demás nutrientes, se modificó su utilización, causando una desnutrición generalizada y cooperando así con el proceso de génesis de la osteoporosis.

La ovariectomía indujo cambios hormonales que favorecieron el proceso de desmineralización condicionante de osteoporosis, validándose así el modelo experimental.

\section{Summary}

34 female, 7-week-old, Sprague-Dawley rats were used to investigate the effect of high-fat diet on postmenopausal osteoporosis. 26 of them were subjected to bilateral oophorectomy. 13 of these were then fed on a high fat diet (group A), the other 13 were fed a normal, regular diet (group B). Eight untreated rats were fed on a normal, regular diet and served as controls.

Five months after their oophorectomy, the hypercalorific diet was found to have diminished the calcium content of lumbar vertebrae (L3, L4 and L5). This study shows that rats fed on a high-fat diet did reduce their food intake and food conversion efficiency. Therefore, this diet induces generalised malnutrition and thus contributes to the proccess of osteoporosis.

Oophorectomy induces endocrinological changes that contribute to the development of osteoporosis. This work leads us to conclude that the oophorectomised rat provides a good experimental model for the study of postmenopausal osteoporosis.

Bacterióloga, Universidad de Los Andes, Santafé de Bogotá, Colombia.

2 M.D., M.Sc., Coordinador de Investigación, Laboratorio de Genética y Metabolismo Mineral, Instituto Nacional de Salud, Santafé de Bogotá, Colombia. 
La osteoporosis es considerada como la reducción de masa ósea por unidad de volumen, debido a que la resorción supera la formación ósea (1).

La osteoporosis es un problema de salud pública a nivel mundial. La mayor parte de los casos se presentan en mujeres posmenopáusicas. Lo anterior se debe a alteraciones hormonales y metabólicas que se producen, mostrando cambios a nivel trabecular, creando zonas de menor resistencia ósea. Entre los factores de riesgo considerados para la osteoporosis se encuentran; predisposición genética, origen caucásico, masa muscular réducida, menopausia temprana, vida sedentaria, consumo reducido de calcio, consumo excesivo de alcohol, proteínas y fosfatos entre otros (2).

El hueso por ser el depósito más grande de calcio que posee el organismo, se ve directamente influido por hormonas que regulan el metabolismo del calcio como la paratohormona (PTH) y la calcitonina. Se ha demostrado que éstas intervienen en la proliferación y diferenciación de las células del hueso (osteoclastos y osteoblastos), recambio óseo, producción de proteínas fijadoras de calcio y en el metabolismo de fósforo. Este último junto con el calcio forman los cristales de hidroxiapatita, el cual es el principal componente de la matriz ósea (3).

Desde principios de 1950 se han utilizado modelos animales para estudiar cambios óseos en la osteoporosis. La mayoría de estos modelos se basan en dietas bajas en calcio (para incrementar la remodelación), ovariectomía (para bajar los niveles de estrógenos) e inmovilización (4). Aunque los perros y primates tienen una remodelación ósea comparable a la de los humanos; éstos no son modelos animales ideales por el costo, la disponibilidad y la dificultad de mantenerlos y experimentar con ellos. Como resultado de estos factores la rata continúa siendo el modelo animal más estudiado en la pérdida del hueso por deficiencia de hormonas ováricas (5). La deficiencia de estrógenos hace que se aumente la pérdida de masa ósea. No se conoce el mecanismo exacto, por el cual los estrógenos elevan los niveles de calcitonina reduciendo así la resorción ósea (2).

En este experimento se logró observar el efecto de la dieta hipercalórica en cuanto a que se disminuye el consumo de otros nutrientes y se modifica la utilización de los mismos, cooperando así con el proceso de osteoporosis.

\section{Materiales y métodos}

Animales y dieta: el proyecto se inició con treinta y cuatro ratas hembra Sprague-Dawley CD-1: CENP INS, de siete semanas de edad. Se mantuvieron en una sala de experimentación en el bioterio central del Instituto Nacional de Salud, bajo las siguientes condiciones: área: $11,31 \mathrm{~m}$, temperatura: $20-24^{\circ} \mathrm{C}$, humedad relativa: $55-65 \%$, iluminación controlada por fotoperiodos de 12 horas, aire filtrado del exterior con una pureza del 100\% (12 cam-bios de aire por hora), ambiente totalmente aislado de ruidos y contaminantes.

Veintiséis ratas se sometieron a gonadectomía bilateral, previa anestesia con inyección de clorhidrato de ketamina y xilazina a dosis de 50 $\mathrm{mg} / \mathrm{kg}$ y $10 \mathrm{mg} / \mathrm{kg}$ de peso. Estas ratas, a su vez, se dividieron en dos grupos, cada uno con trece ratas, quedando conformados los grupos $A$ y $\mathrm{B}$. El grupo control $\mathrm{C}$ quedó constituido por las restantes ocho ratas no gonadectomizadas. Para evaluar la influencia de la dieta hipercalórica en el desarrollo de osteoporosis posmenopáusica, al grupo A se le suministró una dieta rica en grasa, mientras que los grupos B y C recibieron una dieta regular Rodentina ${ }^{\mathrm{R}}$.

La dieta regular de Rodentina, distribuida por Purina S.A, contiene $3,23 \%$ de grasa, $23 \%$ de proteínas, $53,47 \%$ de carbohidratos y $20,30 \%$ de otros, con un total de $3,73 \mathrm{kcal} / \mathrm{g}$.

La dieta hipercalórica se preparó impregnando la dieta regular con aceite vegetal poliinsaturado. La dieta hipercalórica estaba constituida por; $15,7-16,6 \%$ de grasa, $20,5-20,8 \%$ de proteínas, $42,5-43 \%$ de carbohidratos y $20,3 \%$ de otros. El contenido total de kilocalorías por gramo variaba entre 4,34-4,43. 
Los días 56 y 140 de experimentación se obtuvieron muestras por punción del plexo orbital en el saco conjuntival.

\section{Procedimientos analíticos}

Hormona paratiroidea: los niveles de PTH en suero se midieron a través del kit comercial Coat-A-Count Intact PTH IRMA, Diagnostic Products Corporation (DPC), catalogue no. IKPH1. El procedimiento se basa en un ensayo inmunoradiométrico (IRMA).

Calcitonina: el kit comercial empleado para la medición cuantitativa de calcitonina fue: Calcitonin Double Antibody, DPC, catalogue no. KCLD1. El procedimiento se basa en un radioinmunoanálisis (RIA).

Osteocalcina: la osteocalcina se determinó por medio de Radioimmunoassay Kit for the Quantitative Measurement of Osteocalcin (Bone GlaProtein), Diagnostic Systems Laboratories Inc., catalogue no. DSL 6900 . La determinación se basa en un RIA.

Fósforo: el fósforo sérico se midió con el kit comercial de Human Gesellschaft für Biochemica and Diagnostica $\mathrm{mbH}$, Phosphor liquirapid. El fosfato al reaccionar con molibdato en un medio fuertemente ácido, forma un complejo. La absorbancia se lee a $340 \mathrm{~nm}$.

Calcio en vértebras: después de sacrificar las ratas, se extrajo la tercera, cuarta y quinta vértebra lumbar, a las cuales se les quitó todo resto de músculo y grasa.

Los huesos se hidrolizaron a $200^{\circ} \mathrm{C}$ durante un lapso de veinte horas, en $\mathrm{HCl}$ concentrado. Se diluyeron alícuotas del hidrolizado con una solución de lantano al $0,1 \%$, para luego medir el calcio por absorción atómica. Sensibilidad $0,01 \mathrm{ppm}$.

Perfil lipídico: el perfil lipídico comprendió: colesterol total, lipoproteínas de alta densidad (HDL), lipoproteínas de baja densidad (LDL) y triglicéridos.

El colesterol total se midió con el kit Colesterol CHOD-PAP, Diagnostica MERCK, Ref D 010470250.
El colesterol HDL se midió por medio del kit comercial HDL-Cholesterol (CHOD-PAPmethod), reactivo precipitante, Diagnostica MERCK, Ref 14210.

El kit comercial empleado para cuantificar el colesterol LDL fue: $L D L$-Colesterol, reactivo precipitante, Diagnostica MERCK, Ref. A 114992001.

La medición de triglicéridos se hizo con el kit comercial Test colorimétrico totalmente enzimático método GPO-PAP, Diagnostica MERCK, Ref 14354.

Densitometría ósea: el equipo empleado para la realización de este examen fue el OSTEODENSITOMETRO QDR 2000 HOLOGIC. Para la elaboración de la densitometría se escogieron al azar dos ratas de cada uno de los grupos estudiados.

Análisis estadístico: se emplearon los estadísticos T Student y análisis de varianza de una vía (ANOVA), para poblaciones con distribución normal, o Kruskall-Wallis en caso contrario. Las diferencias se consideraron significativas cuando $p<0,05$.

\section{Resultados}

Variación de peso: las ratas presentaron un progresivo aumento de peso durante el tiempo de experimentación. El día inicial no hubo diferencia significativa entre los tres grupos $(\mathrm{P}=\mathrm{NS})$.

En los días experimentales 56 y 140, las ratas gonadectomizadas (grupos A y B) mostraron una mayor ganancia de peso que las ratas control no gonadectomizadas (tabla 1).

Variación de talla: hubo un aumento gradual de la talla durante el curso de la investigación. La talla el día inicial fue similar en los tres grupos ( $\mathrm{P}=\mathrm{NS}$ ). En los días experimentales 56 y 140 las ratas gonadectomizadas presentaron un mayor crecimiento que las ratas no gonadectomizadas (tabla 1).

Consumo de alimento: la media del consumo de alimento (rodentina) en relación al peso ganado en el tiempo de experimentación $(\delta \mathrm{g})^{1} \pm$ desviación estándar fue; grupo A de $20,62 \mathrm{~g} / \delta \mathrm{g}$ 
$\pm 2,88$, grupo B de $25,45 \mathrm{~g} / \delta \mathrm{g} \pm 4,47$, grupo C de $111,94 \mathrm{~g} / \delta \mathrm{g} \pm 20,40$. Las ratas gonadectomizadas necesitaron consumir menos alimento para ganar un gramo de peso que las ratas no gonadectomizadas; $A$ y $B(p<0,05), A y$ $C(p<0,005)$, B y $C(p<0,005)$.

El consumo de lípidos, carbohidratos, proteínas y total de kilocalorías en relación al peso corporal ganado (tabla 2), fueron mayores para el grupo control de ratas no gonadectomizadas.
Hubo diferencia significativa para el consumo de los tres tipos de alimento entre los grupos $\mathrm{A}$ y $B$, ratas gonadectomizadas con dieta hipercalórica y dieta regular, respectivamente; sin embargo, el consumo de kilocalorías por gramo de peso ganado, no fue estadísticamente diferente. Esto es importante en el análisis de resultados, puesto que la dieta hipercalórica no conllevó a aumento en el consumo de alimento, sino que, al contrario, lo disminuyó.

Tabla 1. Variación de peso y talla.

\begin{tabular}{|c|c|c|c|c|c|c|}
\hline & $\begin{array}{c}\text { Grupo A } \\
\text { OVX } \\
\text { DHC } \\
(n=11) \\
\end{array}$ & $\begin{array}{c}\text { Grupo B } \\
\text { OVX } \\
\text { DR } \\
(n=11) \\
\end{array}$ & $\begin{array}{c}\text { Grupo C } \\
\text { NO-OVX } \\
\text { DR } \\
(n=5)\end{array}$ & $\underset{A-B}{P}$ & $\stackrel{P}{\mathbf{P}-\mathrm{C}}$ & $\underset{B-C}{P}$ \\
\hline $\begin{array}{l}\text { Peso } \\
\text { Día } 56 \\
\text { Día } 140\end{array}$ & $\begin{array}{l}382,6 \pm 16,9 \\
401,1 \pm 20,2\end{array}$ & $\begin{array}{l}384 \pm 29,3 \\
401 \pm 29\end{array}$ & $\begin{array}{r}304,08 \pm 10,3 \\
312,9 \pm 8,4\end{array}$ & $\begin{array}{c}\mathrm{a} \\
\mathrm{NS} \\
\mathrm{NS}\end{array}$ & $\begin{array}{c}\mathrm{a} \\
P<0,001 \\
P<0,001\end{array}$ & $\begin{array}{c}a \\
P<0,001 \\
P<0,001\end{array}$ \\
\hline $\begin{array}{l}\text { Talla } \\
\text { Día } 56 \\
\text { Día } 140\end{array}$ & $\begin{array}{l}46,9 \pm 0,5 \\
48,9 \pm 0,5\end{array}$ & $\begin{array}{l}47,2 \pm 0,6 \\
48,9 \pm 0,4\end{array}$ & $\begin{array}{l}45,3 \pm 1 \\
47,5 \pm 0,2\end{array}$ & $\begin{array}{l}\mathrm{b} \\
\text { NS } \\
\text { NS }\end{array}$ & $\begin{array}{c}\text { b } \\
\text { NS } \\
P<0,001\end{array}$ & $\begin{array}{c}\text { b } \\
P<0,01 \\
P<0,001\end{array}$ \\
\hline
\end{tabular}

Todos los valores son la media \pm desviación estándar

a. Determinada por $\mathrm{T}$ Student con un nivel de significancia de $\mathrm{P}<0,05$

b. Determinada por Kruskall-Wallis con un nivel de significancia de $P<0,05$

$\mathrm{NS}=$ no significativo; $\mathrm{OVX}=$ gonadectomizadas; $\mathrm{DHC}=$ dieta hipercalórica; $\mathrm{DR}=$ dieta regular

Tabla 2. Resultados del consumo de alimento.

\begin{tabular}{|c|c|c|c|c|c|c|}
\hline & $\begin{array}{c}\text { Grupo A } \\
\text { OVX } \\
\text { DHC } \\
(n=11) \\
\end{array}$ & $\begin{array}{c}\text { Grupo B } \\
\text { OVX } \\
\text { DR } \\
(n=11)\end{array}$ & $\begin{array}{c}\text { Grupo C } \\
\text { NO-OVX } \\
\text { DR } \\
(n=5) \\
\end{array}$ & $\underset{A-B}{P}$ & $\stackrel{\text { P }}{B-C}$ & $\stackrel{P}{A-C}$ \\
\hline $\begin{array}{l}\text { Alimento } \\
\text { g/día }\end{array}$ & $13,1 \pm 0,93$ & $16,4 \pm 1,03$ & $14,3 \pm 0,71$ & $p<0,001$ & $p<0,001$ & $p<0,01$ \\
\hline \multirow[t]{2}{*}{$\begin{array}{l}\text { Lípidos } \\
\mathrm{g} / \delta \mathrm{g}\end{array}$} & $3,3 \pm 0,46$ & $0,8 \pm 0,13$ & $3,2 \pm 0,8$ & $\begin{array}{c}b \\
p<0,001\end{array}$ & $\begin{array}{c}b \\
p<0,01\end{array}$ & NS \\
\hline & & & & b & b & b \\
\hline \multirow[t]{2}{*}{$\begin{array}{l}\text { Carbohidratos } \\
\mathrm{gr} / \delta \mathrm{g}\end{array}$} & $8,9 \pm 1,29$ & $13,4 \pm 2,33$ & $59,4 \pm 11$ & $p<0,001$ & $p<0,001$ & $p<0,01$ \\
\hline & & & & b & b & b \\
\hline \multirow[t]{2}{*}{$\begin{array}{l}\text { Proteínas } \\
\mathrm{gr} / \delta \mathrm{g}\end{array}$} & $4,3 \pm 0,6$ & $5,9 \pm 1,03$ & $26,3 \pm 4,86$ & $p<0,01$ & $p<0,01$ & $p<0,01$ \\
\hline & & & & b & b & b \\
\hline $\begin{array}{l}\text { Kilocalorias } \\
\mathrm{Kcal} / \delta \mathrm{g}\end{array}$ & $89,3 \pm 12,3$ & $93,8 \pm 16,3$ & $409 \pm 68,6$ & NS & $p<0,01$ & $p<0,01$ \\
\hline
\end{tabular}

Todos los valores son la media \pm desviación estándar

a. Determinada por T Student con un nivel de significancia de $p<0,05$

b. Determinada por Kruskall-Wallis con un nivel de significancia de $p<0,05$

$\mathrm{NS}=$ no significativo; $\mathrm{OVX}=$ gonadectomizadas; $\mathrm{DHC}=$ dieta hipercalórica; $\mathrm{DR}=$ dieta regular 
Calcitonina: el día 56 los promedios de los niveles de calcitonina en los grupos A, B y C fueron muy similares entre sí ( $\mathrm{P}=\mathrm{NS})$.

El día 140 de experimentación, el grupo de ratas no gonadectomizadas (grupo C) presentó los niveles más bajos de calcitonina, mientras que las ratas gonadectomizadas (grupos A y B) tenían valores similares. Al comparar los resultados obtenidos los días experimentales 56 y 140 , se observó un aumento de los niveles sanguíneos de la hormona en el grupo $A$ $(p<0,005)$ y en el grupo $B(p<0,05)$. Por el contrario, la calcitonina sérica para el grupo $\mathrm{C}$ en las dos mediciones fue muy similar (tabla 3).

Hormona paratiroidea: el día 56 las ratas gonadectomizadas sometidas a una dieta hipercalórica (grupo A) tenían mayores niveles sanguíneos de PTH que las ratas mantenidas con la dieta regular. En el día experimental 140, los niveles sanguíneos de PTH fueron muy similares en los tres grupos estudiados $(\mathrm{P}=\mathrm{NS})$. Con los resultados obtenidos los días 56 y 140 , se observó que en los grupos $A(p<0,005), B$ $(p<0,005)$ y $C(p<0,05)$ se presentó un aumento de los niveles sanguíneos de PTH (tabla 3).

Osteocalcina: en el día 56 los promedios de los datos obtenidos en los tres grupos estudiados, no fueron diferentes entre sí. En el día 140 de experimentación, las ratas gonadectomizadas con dieta hipercalórica (grupo A) presentaron los niveles más bajos de la proteína, mientras que las ratas control no gonadectomizadas tenían los niveles más altos. Se encontró diferencia significativa al comparar entre sí los niveles promedio de osteocalcina, obtenidos los días 56 y 140 de experimentación, para el grupo $A(p<0,0005)$ y para el grupo $B$ $(p<0,05)$, presentándose en los dos casos disminución de la proteína. Por el contrario, en las ratas control los niveles de osteocalcina en las

Tabla 3. Resultados de los procedimientos analíticos realizados.

\begin{tabular}{|c|c|c|c|c|c|c|}
\hline & $\begin{array}{c}\text { Grupo A } \\
\text { OVX } \\
\text { DHC }\end{array}$ & $\begin{array}{c}\text { Grupo B } \\
\text { OVX } \\
\text { DR }\end{array}$ & $\begin{array}{c}\text { Grupo C } \\
\text { NO-OVX } \\
\text { DR }\end{array}$ & $\stackrel{P}{\mathbf{P}-\mathrm{B}}$ & $\stackrel{\text { P }}{\text { B-C }}$ & $\underset{A-C}{P}$ \\
\hline Calcitonina & & & & $\mathrm{b}$ & $b$ & $b$ \\
\hline $\begin{array}{l}\text { Día } 56 \\
(n=7)\end{array}$ & $128,6 \pm 65,9$ & $162,08 \pm 8,6$ & $212,6 \pm 122$ & NS & NS & NS \\
\hline $\begin{array}{l}\text { Día } 140 \\
(n=11)\end{array}$ & $345,5 \pm 158$ & $319,8 \pm 185$ & $138,4 \pm 29,2$ & NS & $p<0,05$ & $p<0,001$ \\
\hline PTH & & & & b & $b$ & b \\
\hline & $11,31 \pm 4,7$ & $7,39 \pm 2,9$ & $7,17 \pm 2,2$ & NS & NS & $p<0,05$ \\
\hline $\begin{array}{l}\text { Día 140 } \\
(n=11)\end{array}$ & $19,97 \pm 2,8$ & $28,8 \pm 16,6$ & $31,5 \pm 20,8$ & NS & NS & NS \\
\hline Osteocalcina & & & & b & b & b \\
\hline $\begin{array}{l}\text { Día } 56 \\
(n=7)\end{array}$ & $0,63 \pm 0,09$ & $0,63 \pm 0,18$ & $0,84 \pm 0,32$ & NS & NS & NS \\
\hline $\begin{array}{l}\text { Día } 140 \\
(n=11)\end{array}$ & $0,24 \pm 0,11$ & $0,42 \pm 0,12$ & $0,6 \pm 0,075$ & $p<0,01$ & $p<0,05$ & $p<0,01$ \\
\hline Fósforo & & & & a & a & $\stackrel{a}{a}<001$ \\
\hline $\begin{array}{l}\text { Día 140 } \\
(n=11)\end{array}$ & $4,55 \pm 0,64$ & $5,02 \pm 0,88$ & $5,55 \pm 0,6$ & NS & NS & $p<0,001$ \\
\hline Calcio en vértebras & & & & $\mathrm{a}$ & a & a \\
\hline $\begin{array}{l}\text { Día } 140 \\
(n=11)\end{array}$ & $13.979 \pm 1.092$ & $14.540 \pm 2.160$ & $16.711 \pm 1.900$ & NS & $p<0,05$ & $p<0,01$ \\
\hline
\end{tabular}

Todos los valores son la media \pm desviación estándar.

a. Determinada por T Student con un nivel de significancia de $p<0,05$

b. Determinada por Kruskall-Wallis con un nivel de significancia de $p<0,05$

$\mathrm{NS}=$ no significativo; $\mathrm{OVX}=$ gonadectomizadas; $\mathrm{DHC}=$ dieta hipercalórica; $\mathrm{D} . \mathrm{R}=$ dieta regular 
Tabla 4. Resultados del perfil lipídico.

\begin{tabular}{lcccccc}
\hline & $\begin{array}{c}\text { Grupo A } \\
\text { OVX } \\
\text { DHC } \\
(\mathbf{n = 1 1 )}\end{array}$ & $\begin{array}{c}\text { Grupo B } \\
\text { OVX } \\
\text { DR } \\
(\mathbf{n = 1 1 )}\end{array}$ & $\begin{array}{c}\text { Grupo C } \\
\text { NO-OVX } \\
\text { DR } \\
(\mathbf{n = 5})\end{array}$ & $\begin{array}{c}\mathbf{P}^{*} \\
\mathbf{A - B}\end{array}$ & $\begin{array}{c}\mathbf{P}^{\star} \\
\mathbf{B - C}\end{array}$ & $\begin{array}{c}\mathbf{P}^{\star} \\
\mathbf{A}-\mathbf{C}\end{array}$ \\
\hline $\begin{array}{l}\text { Colesterol } \\
\text { Total mg\% }\end{array}$ & $114,3 \pm 11,4$ & $116,4 \pm 20,1$ & $130,8 \pm 20,4$ & NS & NS & p<0,05 \\
$\begin{array}{l}\text { Colesterol } \\
\text { LDL mg\% }\end{array}$ & $28,3 \pm 14,1$ & $25,4 \pm 14,2$ & $32 \pm 11,4$ & NS & NS & NS \\
$\begin{array}{l}\text { Colesterol } \\
\text { HDL mg\% }\end{array}$ & $64,3 \pm 8,6$ & $61 \pm 7,7$ & $56,7 \pm 11$ & NS & NS & NS \\
$\begin{array}{l}\text { Triglicéridos } \\
\text { mg\% }\end{array}$ & $93 \pm 25,06$ & $104,2 \pm 34,9$ & $134,9 \pm 42,8$ & NS & NS & P<0,05 \\
\hline
\end{tabular}

Todos los valores son la media \pm desviación estándar

*Determinada por T Student con un nivel de significancia de $\mathrm{p}<0,05$

$\mathrm{NS}=$ no significativo; $\mathrm{OVX}=$ gonadectomizadas; $\mathrm{DHC}=$ dieta hipercalórica; $\mathrm{DR}=$ dieta regular

dos mediciones fueron muy similares $(\mathrm{P}=\mathrm{NS})$ (tabla 3).

Fósforo: las ratas gonadectomizadas sometidas a un régimen hipercalórico (grupo A) presentaron los niveles más bajos de fósforo, mientras que las ratas control (grupo C) (no gonadectomizadas con dieta regular) tenían los más altos (tabla 3).

Calcio en vértebras: las ratas gonadectomizadas (grupos $\mathrm{A}$ y $\mathrm{B}$ ) tenían menor concentración de calcio en vértebras lumbares $(\mathrm{L} 3, \mathrm{~L} 4$ y L5), que las ratas control no gonadectomizadas (tabla 3).

Perfil lipídico: el perfil lipídico realizado estaba constituido por: colesterol total, colesterol LDL, colesterol HDL y triglicéridos. Los resultados se muestran en la tabla 4.

Densitometrías: los resultados de las densitometrías (densidad mineral del hueso, densidad mineral lumbar L3, L4, L5 y porcentaje de grasa corporal total) se pueden observar en la tabla 5.

\section{Discusión}

Numerosos estudios han demostrado que la ovariectomía en ratas acelera la pérdida ósea, como consecuencia del déficit estrogénico (1015). Partiendo de este hecho comprobado se gonadectomizaron las ratas, para inducir el desarrollo de osteoporosis posmenopáusica.
Tabla 5. Resultados de las densitometrías.

\begin{tabular}{lccc}
\hline & $\begin{array}{c}\text { Grupo A } \\
\text { OVX } \\
\text { DHC } \\
(\mathbf{n}=2)\end{array}$ & $\begin{array}{c}\text { Grupo B } \\
\text { OVX } \\
\text { DR } \\
(\mathbf{n}=2)\end{array}$ & $\begin{array}{c}\text { Grupo C } \\
\text { NO-OVX } \\
\text { DR } \\
(\mathbf{n}=2)\end{array}$ \\
\hline BMD & $0,1588 \pm 0,01$ & $0,160 \pm 0,006$ & $0,157 \pm 0,004$ \\
BMD & $0,191 \pm 0,009$ & $0,184 \pm 0,001$ & $0,205 \pm 0,01$ \\
Lumbar & $7,5 \pm 2$ & $6,75 \pm 0,15$ & $8,05 \pm 1,35$ \\
$\%$ Grasa & & & \\
\hline global & &
\end{tabular}

Todos los valores son la media \pm desviación estándar $\mathrm{BMD}=$ Densidad media del hueso; $\mathrm{OVX}=$ Gonadectomizadas; $\mathrm{DHC}=$ Dieta hipercalórica; $\mathrm{DR}=$ Dieta regular

Las ratas gonadectomizadas presentaron mayor ganancia de peso que las ratas no gonadectomizadas (control). Este fenómeno ha sido reportado por varios investigadores $(5,12$, 13). Las ratas gonadectomizadas (grupos A y B) experimentaron mayor crecimiento. Lo anterior puede ser el resultado del desbalance metabólico secundario al déficit estrogénico, planteado para el aumento de la hormona del crecimiento, causado por el descontrol de la actividad hipotalámica. También, al disminuir los estrógenos circulantes, el cierre de las epífisis se retarda, dando tiempo a que el hueso alcance un tamaño mayor $(13,16)$. La ovariectomía incrementa el crecimiento longitudinal y del periostio del hueso, pero, reduce la formación endosteal $(13,17)$. 
El objetivo del estudio era determinar la influencia de la dieta hipercalórica en el desarrollo de osteoporosis posmenopáusica, para lo cual se aumentó la proporción de lípidos en la dieta mediante la utilización de un aceite vegetal poliinsaturado. Se logró un alimento de alto valor calórico, $15 \%$ superior a la dieta regular, aceptable para las ratas del grupo $\mathrm{A}$.

El consumo de alimento fue menor en las ratas sometidas a la dieta hipercalórica (grupo A) y, a pesar, de la mayor cantidad de kilocalorías, con respecto al tipo de alimento regular de los grupos B y C, el consumo de kilocalorías fue similar en los tres grupos, pero no así la utilización, es decir la eficiencia del alimento, puesto que en las ratas gonadectomizadas (grupos $\mathrm{A}$ y $\mathrm{B}$ ), las calorías necesarias para ganar un gramo de peso corporal, son mucho menores que para lograr el mismo efecto en las ratas control no gonadectomizadas, puntualizando el efecto metabólico de la gonadectomía.

Sin embargo, debido posiblemente al alto valor energético del alimento del grupo A, se alcanza más rápidamente el nivel de saciedad por parte del animal, reduciendo significativamente el consumo de alimento por debajo de las ratas gonadectomizadas mantenidas con una dieta regular (grupo B), condicionando un estado de desnutrición generalizada, ya que al aumentar el porcentaje lipídico de la dieta se disminuye el contenido de otros requerimientos nutricionales, entre los cuales están: proteínas, carbohidratos, minerales y vitaminas. Desde este punto de vista, se logró observar el efecto de una dieta hipercalórica, en cuanto a que disminuye la ingesta de los demás nutrientes y modifica su utilización, pero, no se logró evidenciar el efecto de un exceso de calorías, manteniendo constantes los demás parámetros nutricionales.

\section{PTH y calcitonina}

En los resultados obtenidos el día 56 , las ratas gonadectomizadas y sometidas a la dieta hipercalórica, presentaron los niveles más altos de PTH, lo cual podría deberse no sólo a la rápida pérdida ósea inducida por la gonadectomía $(14,17)$, sino también al hecho de que las dietas con alto contenido lipídico disminuyen la biodisponibilidad del calcio (18). Las ratas no gonadectomizadas con dieta regular tenían niveles más altos que el grupo control, aunque esta diferencia no fue significativa. En este caso, podría sugerirse que la dieta en el grupo A, contribuiría al daño óseo postgonadectomía.

El día 140, los niveles de calcitonina de las ratas gonadectomizadas fueron mayores a los presentados el día 56 , lo anterior podría ser el resultado de un periodo relativo de estabilización de la pérdida de hueso trabecular, evitando así la pérdida exagerada de calcio.

\section{Osteocalcina}

La pérdida de hueso inmediatamente después de la menopausia, puede ser evidenciada por altos niveles de marcadores de recambio óseo, como la osteocalcina. El día 56 no se observó una clara diferencia entre los tres grupos, ya que todos presentaban niveles similares de esta proteína no colágena. El día 140, el grupo C presentó los niveles más altos de osteocalcina y hubo diferencia significativa con los otros grupos. Estos resultados son contradictorios con los esperados, puesto que se sabe que los niveles de osteocalcina aumentan en estados de alto recambio óseo, como sería el caso para las ratas gonadectomizadas (1921).

No fue posible emplear un RIA (radioinmunoensayo) que detectara específicamente la osteocalcina de rata, produciendo valores que realmente no corresponden. Se informan pero no se les da valor concluyente.

\section{Calcio en vértebras lumbares}

En los resultados que se obtuvieron en la medición de calcio en vértebras, se observó que los mayores niveles de calcio se encontraban en las ratas no gonadectomizadas (grupo C) y los más bajos en las ratas gonadectomizadas (grupos A y B). Cabe anotar que el grupo A tiene menor cantidad de calcio que el grupo B aunque esta diferencia no es significativa. Estos resultados corresponden con lo esperado para la pérdida de hueso tra- 
becular en consecuencia a la gonadectomía. No se evidenció el efecto de la dieta.

\section{Densitometría}

Se consideró el promedio para la pareja de cada grupo, teniendo presente que el valor no es representativo estadísticamente, pero provee una información útil para experimentos posteriores.

La densidad fue similar para los tres grupos, así como la proporción de grasa corporal. Puesto que el método es muy sensible, preciso y, además, indica el contenido de calcio del hueso, se podría interpretar como número insuficiente de casos estudiados, pero también debe analizarse la posibilidad de que la densitometría en región lumbar para rata, presente dificultades de estandarización.

\section{Conclusiones}

Se logró observar el efecto de la dieta hipercalórica en cuanto a que disminuye la ingesta de los demás nutrientes y se modifica su utilización, causando una desnutrición generalizada y cooperando así con el proceso de génesis de la osteoporosis. Se ha demostrado en este estudio que la gonadectomía induce cambios hormonales que favorecen el proceso de desmineralización condicionante de osteoporosis, validando así el modelo experimental. Además de los cambios que se producen por la gonadectomía, se observó la influencia de la dieta, en los marcadores de remodelación ósea.

Se recomienda para futuros estudios evaluar con densitometría ósea grupos más amplios, como también utilizar otros marcadores más específicos comocross linksy calcio urinario para lo cual se hace necesario la utilización de jaulas metabólicas. Para posteriores estudios donde se determinen los niveles séricos de osteocalcina se aconseja emplear un kit que detecte específicamente la osteocalcina de rata.

\section{Agradecimientos}

Al bioterio de experimentación del Instituto Nacional de Salud. Al doctor Antonio Niño del Centro de Endocrinología y Enfermedades
Metabólicas por hacer posible las densitometrías. Al Departamento de Química de la Universidad de los Andes por el préstamo del equipo de absorción atómica y la asesoría en la medición de calcio. A los doctores Antonio Iglesias y Miguel Suárez por las correcciones y evaluación de este trabajo como tesis de grado de las autoras.

\section{Referencias}

1. Rico E. Resultado en el tratamiento de fracturas transtrocacétricas con clavo condilocefálico tipo Kuntsher de pacientes seniles con osteoporosis. Universidad Nacional Autónoma de México, Facultad de Medicina 1989;1-4,10-2.

2. Eschere J, Gambert E. Osteoporosis: tratamiento. Mundo Médico 1992;8:37-48.

3. Rubbin E. Patología. Segunda edición. Buenos Aires: Editorial Médica Panamericana, 1990:1174-210.

4. Boskey A. Bone mineral and matriz. Are they altered in osteoporosis? The Orthopedic Clinics of North America 1990;21:19-29.

5. Kalu D, Liu C, Hardin R, Hollis B. The aged rat model of ovarian hormone deficiency bone loss. Endocrinology 1988;124:7-16.

6. Hernandez L, Osorio A. Inmunoanálisis. Fundamentos y aplicaciones. Bogotá: Laboratorio Endocrino Vélez, 1990:35-39.

7. Yalow R, Berson S. Introduction and general considerations. In: Odell W, Daughaday W, editors. Principles of competitive protein binding assays. Filadelfia, 1971.

8. Sokolnikov A, Kodentsova V. The calcium and phosphorus content of the bone tissue in ovariectomized rats with a fracture of the tibia: the effect of vitamin $\mathrm{K}$ and vitamin D metabolites. Fiziol-zh 1992;38:29-36.

9. Miller S, Wronski T. Longterm osteopenic changes in cancellous bone structure in ovariectomized rats. Anat Rec 1993;236:433-41.

10. Schot C, Schuurs W. Pathophysiology of bone loss in castrated animals. J Steroid Biochem Bio 1990;37:461-5.

11. Finkelman R, Bell N, Strong D, Demers L. Ovariectomy selectively reduces the concentration of transforming growth factor $B$ in rat bone:implications for estrogen deficiency-associated bone loss. Proc Natl Acad Sci 1992;89:12190-3.

12. Wronski J, Schenck A, Cintrón M, Walsh C. Effect of body weight on osteopenia in ovariectomized rats. Calcified Tissue International 1987;40:155-9.

13. Wronski J, Lowry L, Walsh C, Ignaszewski A. Skeletal alterations in ovariectomized rats. Calcified Tissue International 1985;3:324-8. 
14. Wronski J, Cintrón M, Dann M. Temporal relationship between bone loss and increased bone turnover in ovariectomized rats. Calcified Tissue International 1988;43:179-83.

15. Mazzuoli G, Minisola S, Blanchi G, Romagnol R, Pasitti T. The effects of oophorectomy on skeletal metabolism. J Steroid Biochem Molec Biol 1990;37:457-9.

16. Moreno E, Fernandez G, Lopez M. Diagnóstico y tratamiento en endocrinología. Madrid: Ediciones Díaz de Santos, 1994:392-5.

17. Wronski J, Dann M, Scott S, Cintrón M. Long-term effects of ovariectomy and aging on the rat skeleton. Calcified Tissue International 1989;45:360-6.
18. Levine B, Einhorn T, Michel P. Nutrition and bone. The orthopedic clinics of North America 1990;21:4350.

19. Greig F, Casas J, Castells S. Changes in plasma osteocalcin concentrations during treatment of rickets. J Pediatr 1989;820-3.

20. Hosoda K, Hiroshi E, Tadakatsu N, Kubota T. Sandwich immunoassay for intact human osteocalcin. Clinical Chemistry 1992;38:2233-8.

21. Brown J, Malaval L, Delmas P, Edouard C. Serum bone gla-protein: a specific marker for bone formation in postmenopausal osteoporosis. Lancet 1984; 19:1091-3. 